\title{
Detection of Polyphase Codes Radar Signals in Low SNR
}

\author{
Runlan Tian, ${ }^{1,2}$ Guoyi Zhang, ${ }^{2}$ Rui Zhou, ${ }^{2}$ and Wei Dong ${ }^{1}$ \\ ${ }^{1}$ College of Electronic Science and Engineering, Jilin University, Changchun 130021, China \\ ${ }^{2}$ Department of Electronic Countermeasure, Aviation University of Air Force, Changchun 130022, China
}

Correspondence should be addressed to Wei Dong; dongweijl@163.com

Received 16 October 2015; Accepted 17 January 2016

Academic Editor: Kishin Sadarangani

Copyright (C) 2016 Runlan Tian et al. This is an open access article distributed under the Creative Commons Attribution License, which permits unrestricted use, distribution, and reproduction in any medium, provided the original work is properly cited.

\begin{abstract}
A novel effective detection method is proposed for electronic intelligence (ELINT) systems detecting polyphase codes radar signal in the low signal-to-noise ratio (SNR) scenario. The core idea of the proposed method is first to calculate the time-frequency distribution of polyphase codes radar signals via Wigner-Ville distribution (WVD); then the modified Hough transform (HT) is employed to cumulate all the energy of WVD's ridges effectively to achieve signal detection. Compared with the generalised Wigner Hough transform (GWHT) method, the proposed method has a superior performance in low SNR and is not sensitive to the code type. Simulation results verify the validity of the proposed method.
\end{abstract}

\section{Introduction}

Modern radar systems face a variety of threats in electronic support system, radar warning receivers, and electronic attack system. In order to survive, the low probability of interception (LPI) signals is employed by modern radar system. Those LPI signals are typically pulse compression continuous signals, which are difficult to intercept by the electronic intelligence (ELINT) systems. Polyphase codes radar signal (Frank, P1, P2, P3, and P4 codes), which derived from step frequency modulated signal and linear frequency modulated signal, is the most frequently used LPI signal because of its easy digital implementation, its versatility, and its high range resolution and Doppler tolerance [1-6]. In the past two decades, the detection of polyphase codes radar signals has attracted much attention [7-13]. The polyphase codes radar signals exhibit the characteristic of multiple ridges parallel in the time-frequency distribution. According to this characteristic, a variety of methods is proposed for detecting the polyphase codes radar signals. Gulum et al. analyzed the signal detection method using Wigner-Ville distributionRadon transform [14]. In [15], the Radon-ambiguity transform was employed to detect polyphase codes radar signals. The fractional autocorrelation (FA) and fractional Fourier transform (FrFT) were considered to solve the problem of polyphase code signal detection in [16] and [17], respectively.
Since all of these methods detect the polyphase codes radar signals using the energy of main ridge in time-frequency distribution, they have two drawbacks as follows: (i) signal detection cannot be achieved in the low signal-to-noise ratio (SNR); (ii) these methods have different performance to different code type, which is not suitable for the ELINT systems.

To make use of all the parallel ridges' energy of polyphase codes radar signal in time-frequency distribution, this Letter derives modified Wigner Hough transform (MWHT), based on which a novel detection method is also proposed. The novel method overcomes the two drawbacks as above. Simulation experiments have been carried out to demonstrate the effectiveness of the novel method.

\section{Proposed Method}

The polyphase codes radar signals can be expressed as

$$
x[n]=A e^{j\left(2 \pi f_{1} n+\phi_{k}\right)}, \quad 0 \leq n \leq N-1,
$$

where $A$ is the amplitude, $f_{1}$ is the carrier frequency, $N$ is the signal length, and $\phi_{k}$ is the phase modulated function. Different phase modulated function represents the polyphase codes radar signal which has different code types. Among them, the Frank, P1, and P2 codes are derived from the step 
frequency modulated signal, while the P3 and P4 codes are derived from linear frequency modulated signal.

The discrete Wigner-Ville distribution (WVD) can be written as

$$
\begin{aligned}
W_{x}[n, f] & =2 \sum_{k=-\infty}^{\infty} C_{x x}[n, k] F[f, k], \\
C_{x x}[n, k] & =x[n+k] x^{*}[n-k], \\
F[f, k] & =e^{-j 4 \pi f k},
\end{aligned}
$$

where $C_{x x}[n, k]$ is the instantaneous time autocorrelation and $F[f, k]$ is the kernel function of the WVD. That is, the WVD can be considered as the Fourier transform of the instantaneous time autocorrelation.

The characteristic of multiple ridges parallel can be obtained by calculating the WVD of the polyphase codes radar signals. Among the multiple ridges, the main ridge occupies the biggest energy. Different code type signal has different energy distribution of ridges. The polyphase codes radar signal detection can be achieved by extracting the ridge in the WVD.

Hough transform (HT) is an effective tool to detect the ridge characteristic in the WVD. Discrete Wigner Hough transform (WHT) can be expressed as

$$
\operatorname{WHT}_{x}[\tilde{f}, \tilde{g}]=\sum_{n} \sum_{k} C_{x x}[n, k] F[\tilde{f}+\tilde{g} n, k],
$$

where $F[\tilde{f}+\tilde{g} n, k]$ is the kernel function of the WHT and $\tilde{f}$ and $\tilde{g}$ denote the frequency and the slope of the ridge in the WVD, respectively. Corresponding to a ridge in WVD, a peak will be revealed in the WHT of polyphase codes radar signal.

Since the characteristic of polyphase codes radar signals in WVD is multiple ridges parallel, the WHT of polyphase codes radar signals has multipeaks, which would result in signal energy dispersion. The energy dispersion is the essential reason of two drawbacks in Section 1. Figure 1 displays the generalised Wigner Hough transform (GWHT) Frank codes radar signals. The GWHT of noiseless polyphase codes radar signals is displayed in Figure 1(a) and reveals several prominent peaks, yet the prominent peak disappears in Figure $1(\mathrm{~b})$ which is a noisy version signal with $\mathrm{SNR}=-6 \mathrm{~dB}$. In order to sufficiently utilize the multiple ridges parallel characteristic in WVD, a novel kernel function which can be used for accumulating all ridges in WVD was proposed as follows.

The complexity of the polyphase codes radar signals prevents an analytical expression of the polyphase codes radar signals' WVD. Nevertheless, based on the characteristic of polyphase codes radar signals in WVD, the expression of WVD and instantaneous time autocorrelation can be constructed as follows:

$$
\begin{aligned}
& C_{s s}[n, k]=\sum_{i} A_{i}^{2} e^{j 4 \pi k\left[\left(f_{1}-i d\right)+g_{1} n\right]}, \\
& W_{s}[n, f]=\sum_{i} \sum_{k=-\infty}^{\infty} A_{i}^{2} e^{-j 4 \pi k\left[f-\left(f_{1}-i d\right)-g_{1} n\right]},
\end{aligned}
$$

where $d$ is the distance between the ridges in WVD, $g_{1}$ is the slope of the ridges in WVD, and $A_{i}^{2}$ denotes the energy of $i$ th ridge. Although (5) is not the exact expression of polyphase codes radar signals' WVD, there will be no influence on the subsequent processing result. The signal energy and the ridge energy have the relationship $A^{2}=\sum_{i} A_{i}^{2}$.

The novel kernel function of the WHT is defined as

$$
F[\tilde{f}-i \tilde{d}+\tilde{g} n, k]=e^{-j 4 \pi k(\tilde{f}-i \tilde{d}+\tilde{g} n \Delta)} .
$$

Thus the MWHT of polyphase code radar signals is

$$
\begin{aligned}
& \operatorname{MWHT}_{s}[\tilde{f}, \tilde{g}, \tilde{d}] \\
& =\sum_{i} \sum_{n} \sum_{k} A_{i}^{2} e^{-j 4 \pi k\left[\left(\tilde{f}-f_{1}\right)+i(\tilde{d}-d)+n\left(\tilde{g}-g_{1}\right)\right]}
\end{aligned}
$$

and is maximized only when evaluated at $\tilde{f}=f_{1}, \widetilde{g}=g_{1}$ and $\widetilde{d}=d$, which results in

$$
\operatorname{MWHT}_{s}\left[f_{1}, g_{1}, d\right]=\frac{N^{2} A^{2}}{2} \text {. }
$$

Equation (8) indicates that the detection performance of MWHT is only loss $3 \mathrm{~dB}$ compared with the matched filter (MF). Figure 2 is the MWHT of Frank code radar signal in different SNR, respectively. The noiseless version and the noisy version signal with $\mathrm{SNR}=-6 \mathrm{~dB}$ both reveal prominent peak which can be used to achieve polyphase codes radar signals detection. The other type polyphase codes radar signals have similar detection performance to the Frank code.

\section{Simulation Results}

To evaluate the effectiveness of the proposed method, we applied it to the simulated polyphase codes radar signals. Simulation parameters are as follows. The carrier frequency is $f_{c}=10 \mathrm{MHz}$, the sampling rate is $f_{s}=70 \mathrm{MHz}$, and the code rate is $t_{b}=0.1 \mu \mathrm{s}$. We ran the simulations 1000 times for each SNR. The detection performance of GWHT, MWHT, and power averaging method (PA) is shown using a receiver operating characteristic (ROC) plotted in Figure 3. The probability of detecting the five type polyphase codes radar signals using MWHT is compared with the same metric using the GWHT and the PA as probability of false alarm is $10^{-2}$.

The detection performance of the GWHT is different for the signal which has different code type, because different code type has different main ridge line energy in the WVD plane. The main ridge energy ratio relationship between the polyphase code signal and the LFM signal with identical signal energy is given by a large number simulation. Table 1 shows the ratio relationship. These results show that the main ridge energy value for $\mathrm{P} 1, \mathrm{P} 2$, and $\mathrm{P} 4$ code reduces by about $25 \%$ relative to the LFM. Then, the main ridge energy values for the Frank and P3 code are over 50\% smaller than the LFM. Among the detection performances of the GWHT method in Figure 3, the P4 code signal 


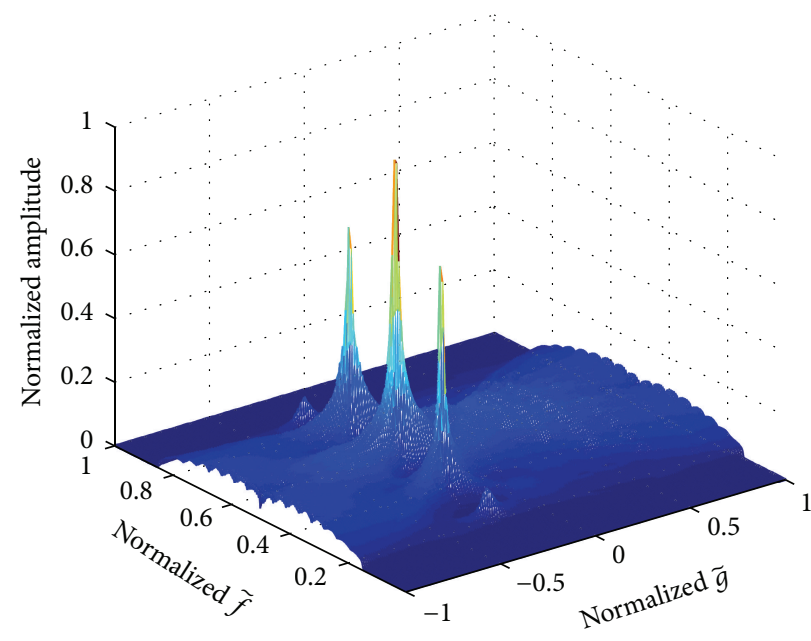

(a) The GWHT of noiseless Frank code

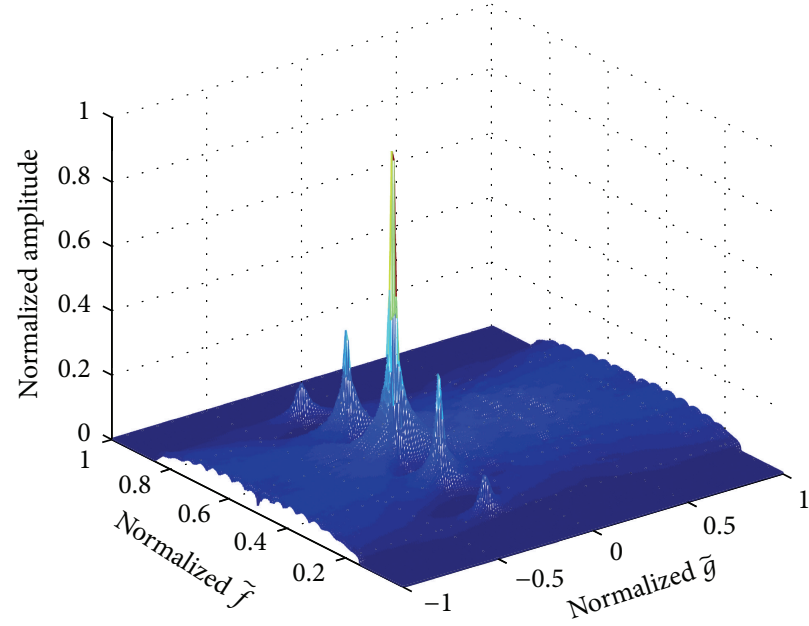

(c) The GWHT of noiseless P1 code

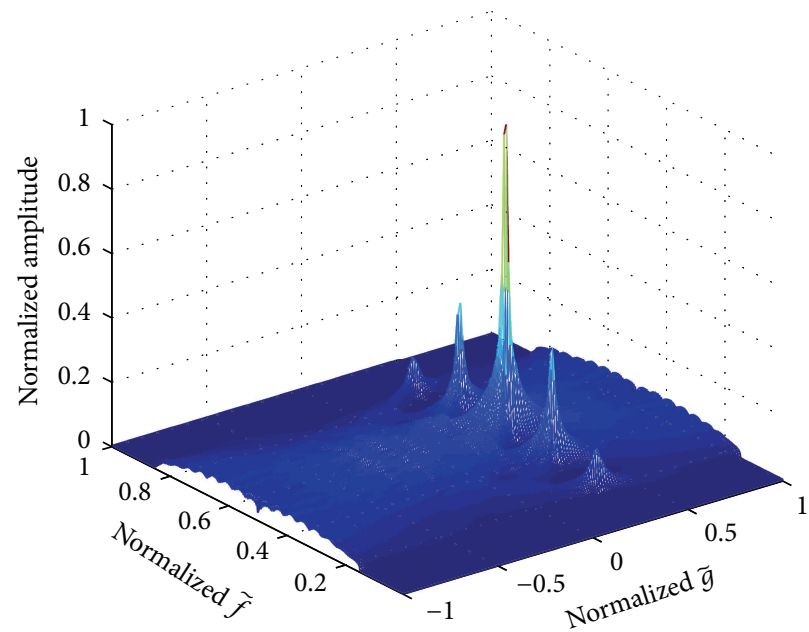

(e) The GWHT of noiseless P2 code

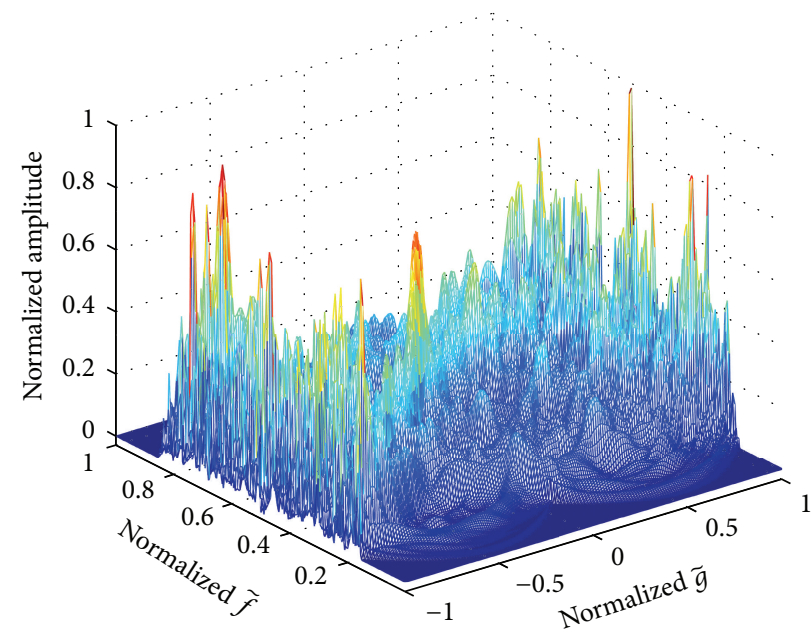

(b) The GWHT of noisy version Frank code

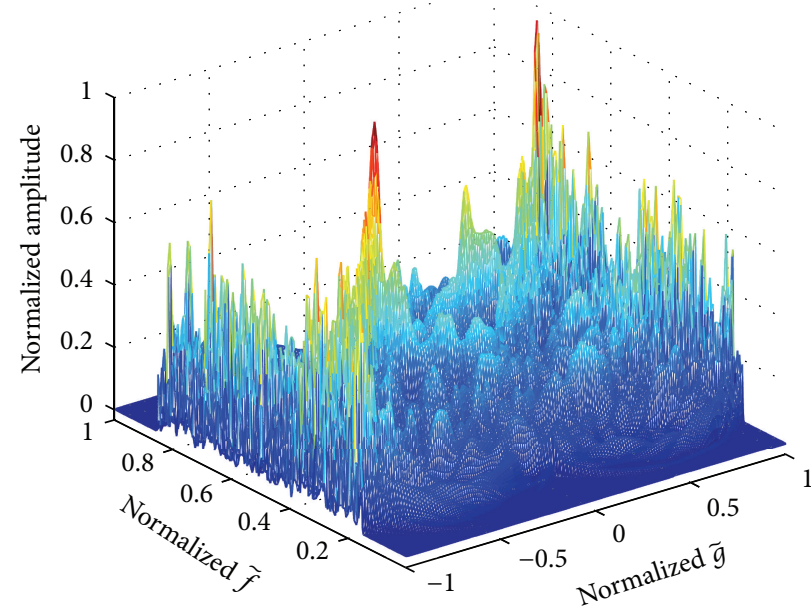

(d) The GWHT of noisy version P1 code

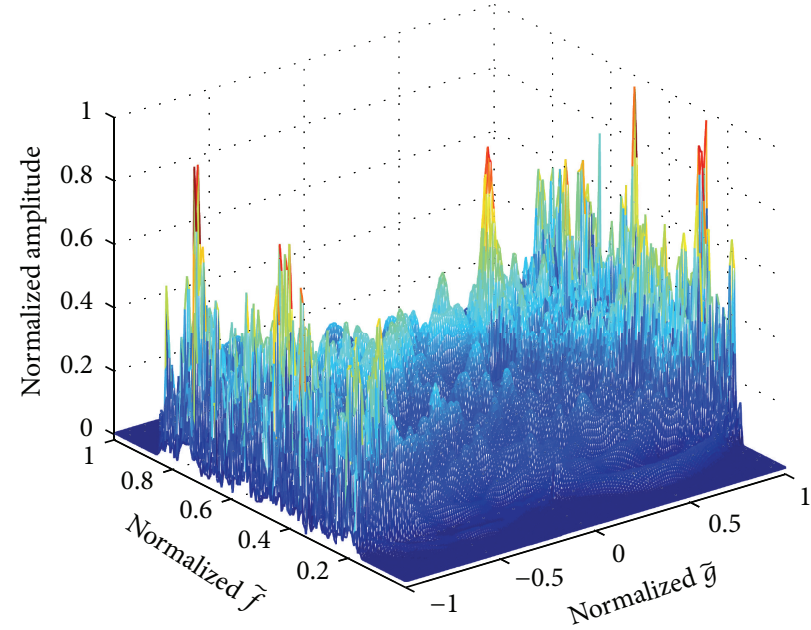

(f) The GWHT of noisy version P2 code

Figure 1: Continued. 


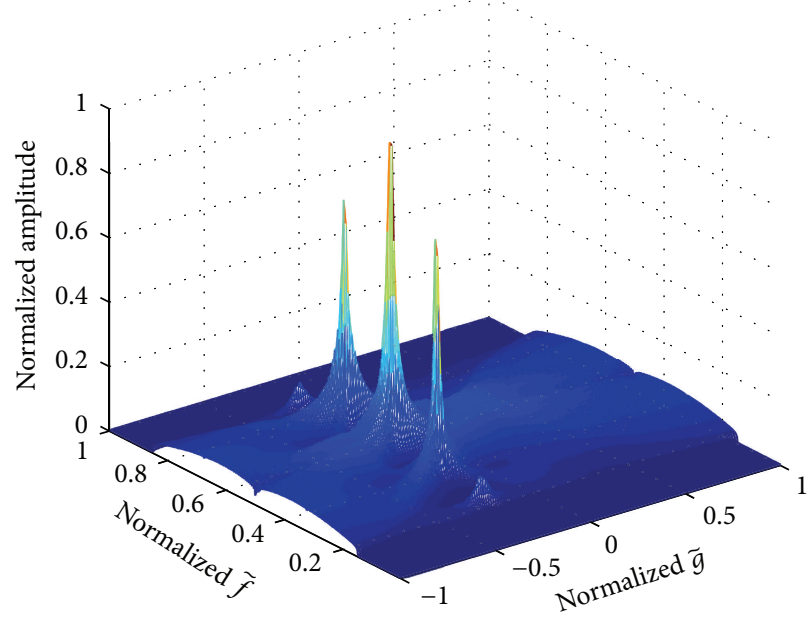

(g) The GWHT of noiseless P3 code

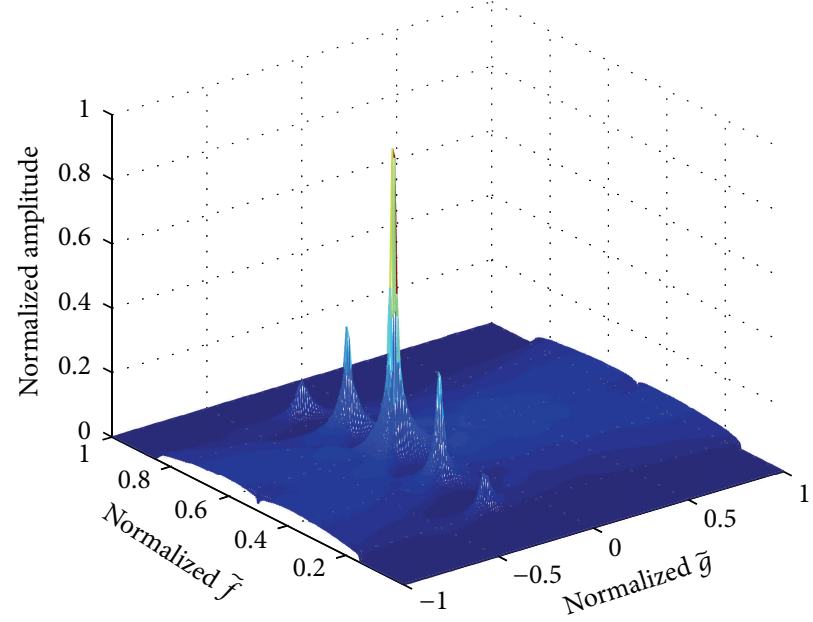

(i) The GWHT of noiseless P3 code

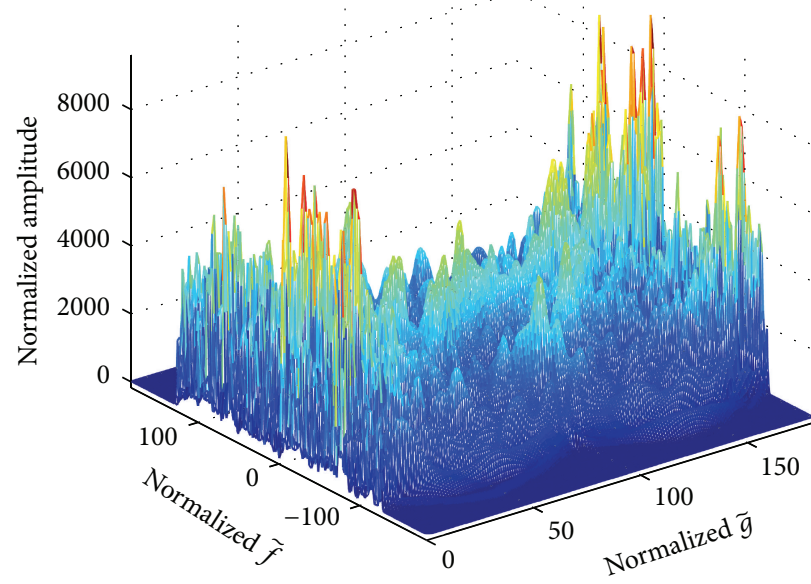

(h) The GWHT of noisy version P3 code

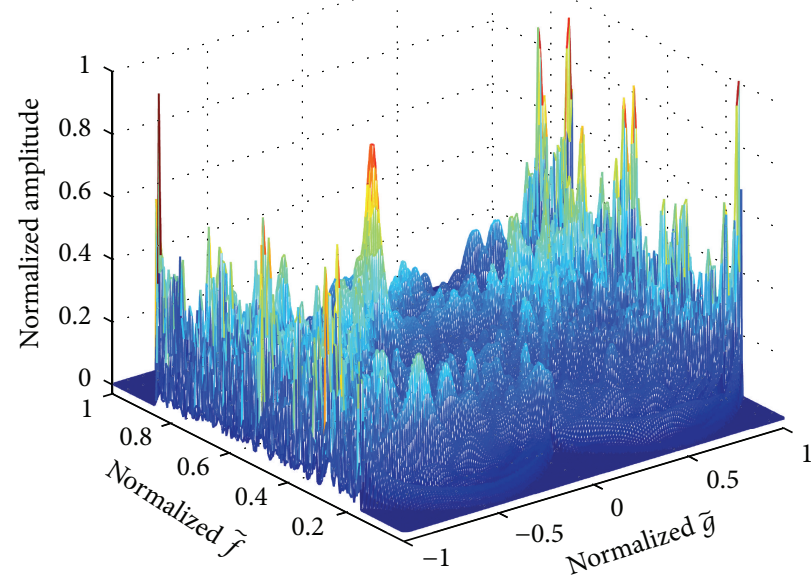

(j) The GWHT of noisy version P3 code

Figure 1: The GWHT of Frank code.

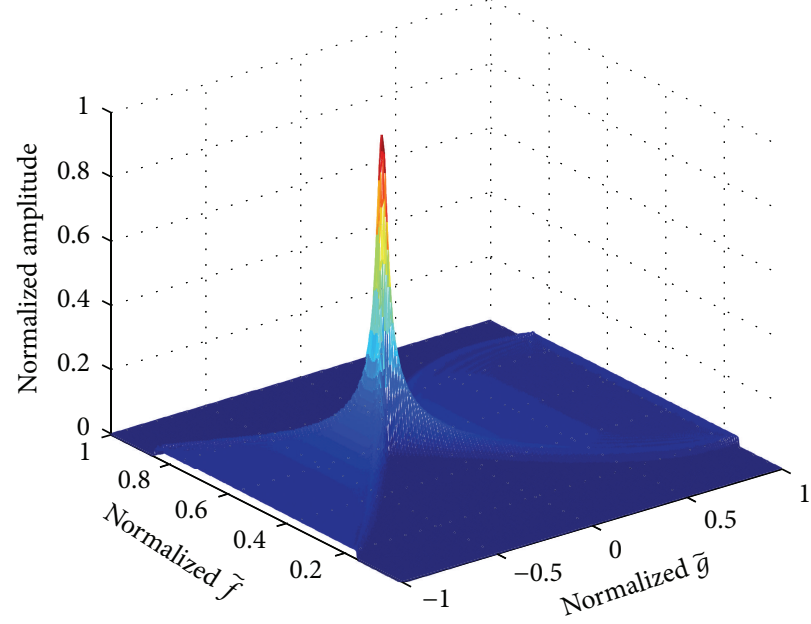

(a) The MWHT of noiseless Frank code

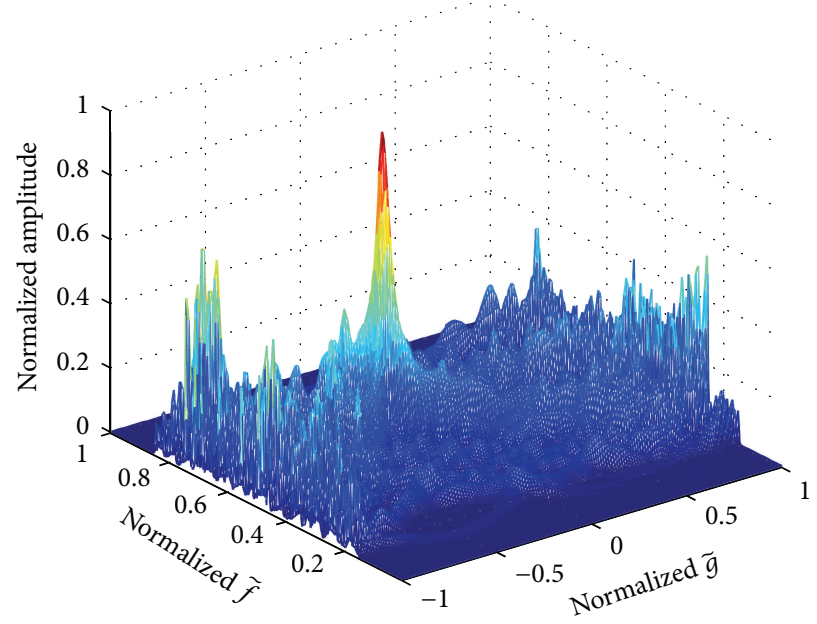

(b) The MWHT of noisy version Frank code

Figure 2: The GWHT of Frank code. 


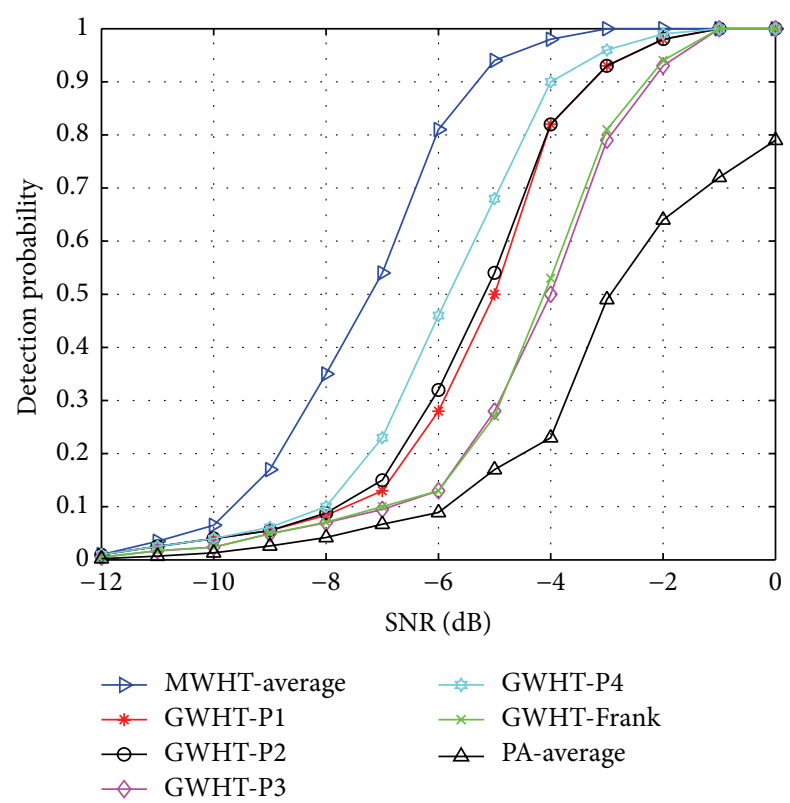

FIGURE 3: Detection performance comparison.

TABLE 1: The ratio relationship of main ridge energy.

\begin{tabular}{lcccccc}
\hline Signal & LFM & Frank & P1 & P2 & P3 & P4 \\
\hline $\begin{array}{l}\text { The energy of } \\
\text { main ridge }\end{array}$ & $E$ & $0.44 E$ & $0.72 E$ & $0.73 E$ & $0.44 E$ & $0.77 E$ \\
\hline
\end{tabular}

had the best detection performance; the following was P1 and P2 code. The detection performance of Frank and P3 code was the worst. The rank relationship of detection performance was consistent with the main ridge energy ratio relationship in Table 1. To make the figure clear enough, the detection performance of the MWHT and the PA method was plotted in average curve since they are almost identical. Simulation results show that the detection performance of PA method is inferior to the WHT based method and the detection performance of MWHT is superior to the GWHT method.

\section{Conclusion}

A novel effective method for polyphase codes radar signals detection is proposed in this Letter. Based on the characteristic of polyphase codes radar signal in WVD, a novel kernel function of the WHT is defined and applied for accumulating all the energy of ridges. Simulation results show that the proposed method can achieve polyphase codes radar signal detection in the low SNR scenario and is not sensitive to the code type, which is needful for ELINT system. In addition, discussion within this Letter is also suitable for other polyphase codes radar signals detection methods based on the time-frequency distribution.

\section{Conflict of Interests}

The authors declare that there is no conflict of interests regarding the publication of this paper.

\section{Acknowledgment}

This work was supported by the National Natural Science Foundation of China (Grant no. 41476089).

\section{References}

[1] T. Felhauer, "New class of polyphase pulse compression code with unique characteristics," Electronics Letters, vol. 28, no. 8, pp. 769-771, 1992.

[2] L. Li, H.-B. Ji, and L. Jiang, "Quadratic time-frequency analysis and sequential recognition for specific emitter identification," IET Signal Processing, vol. 5, no. 6, pp. 568-574, 2011.

[3] P. Camuso, G. Foglia, and D. A. Pistoia, "A comprehensive analysis on detection performances of LPI signals filtering strategies," in Proceedings of the IEEE European Radar Conference (EuRAD '09), pp. 125-128, IEEE, Rome, Italy, SeptemberOctober 2009.

[4] F. Millioz and M. Davies, "Sparse detection in the chirplet transform: application to FMCW radar signals," IEEE Transactions on Signal Processing, vol. 60, no. 6, pp. 2800-2813, 2012.

[5] M. Malanowski and K. Kulpa, "Detection of moving targets with continuous-wave noise radar: theory and measurements," IEEE Transactions on Geoscience and Remote Sensing, vol. 50, no. 9, pp. 3502-3509, 2012.

[6] M. A. Govoni, H. B. Li, and J. A. Kosinski, "Low probability of interception of an advanced noise radar waveform with linearFM," IEEE Transactions on Aerospace and Electronic Systems, vol. 49, no. 2, pp. 1351-1356, 2013.

[7] Z. K. Zhang, J. H. Zhu, Y. B. Tian, and H. L. Li, "Novel sensor selection strategy for LPI based on an improved IMMPF tracking method," Journal of Systems Engineering and Electronics, vol. 25, no. 6, pp. 1004-1010, 2014.

[8] H. Deng, "Polyphase code design for orthogonal netted radar systems," IEEE Transactions on Signal Processing, vol. 52, no. 11, pp. 3126-3135, 2004.

[9] M. Parlak, M. Matsuo, and J. F. Buckwalter, "Analog signal processing for pulse compression radar in 90-nm CMOS," IEEE Transactions on Microwave Theory and Techniques, vol. 60, no. 12, pp. 3810-3822, 2012.

[10] X. N. Zeng, Y. S. Zhang, and Y. D. Guo, "Polyphase coded signal design for MIMO radar using MO-MicPSO," Journal of Systems Engineering and Electronics, vol. 22, no. 3, pp. 381-386, 2011.

[11] L. Anjaneyulu, N. S. Murthy, and N. V. S. N. Sarma, "Identification of LPI radar signals by higher order spectra and neural network techniques," in Proceedings of the International Conference on Electronic Design (ICED '08), pp. 1-6, IEEE, Penang, Malaysia, December 2008.

[12] C. Fancey and C. M. Alabaster, "The metrication of low probability of intercept waveforms," in Proceedings of the 5th International Waveform Diversity and Design Conference (WDD '10), pp. 58-62, Niagara Falls, Canada, August 2010.

[13] N. Paepolshiri, P. E. Pace, and D. C. Jenn, "Extending the unambiguous range of polyphase $\mathrm{P} 4 \mathrm{CW}$ radar using the robust symmetrical number system," IET Radar, Sonar \& Navigation, vol. 6, no. 7, pp. 659-667, 2012.

[14] T. O. Gulum, P. E. Pace, and R. Cristi, "Extraction of polyphase radar modulation parameters using a Wigner-Ville distribution-Radon transform," in Proceedings of the IEEE International Conference on Acoustics, Speech and Signal Processing (ICASSP '08), pp. 1505-1508, IEEE, Las Vegas, Nev, USA, April 2008. 
[15] B. K. Jennison, "Detection of polyphase pulse compression waveforms using the Radon-ambiguity transform," IEEE Transactions on Aerospace and Electronic Systems, vol. 39, no. 1, pp. 335-343, 2003.

[16] D. J. Peacock and B. Santhanam, "Comparison of Centered Discrete Fractional Fourier Transforms for chirp parameter estimation," in Proceedings of the IEEE Digital Signal Processing and Signal Processing Education Meeting (DSP/SPE '13), pp. 6568, Napa, Calif, USA, August 2013.

[17] O. Akay and E. Erözden, "Employing fractional autocorrelation for fast detection and sweep rate estimation of pulse compression radar waveforms," Signal Processing, vol. 89, no. 12, pp. 2479-2489, 2009. 


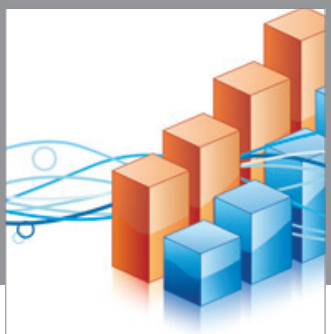

Advances in

Operations Research

vatem alat4

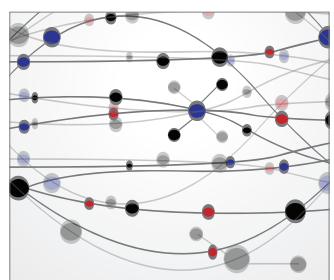

\section{The Scientific} World Journal
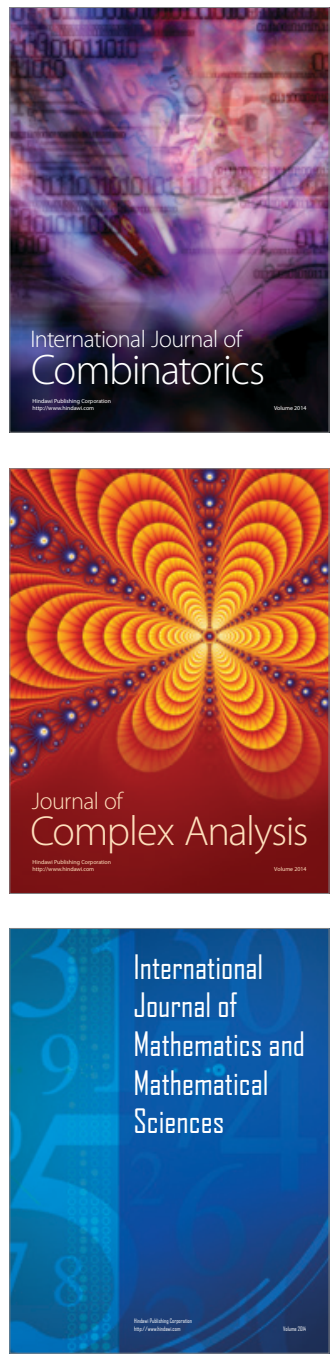
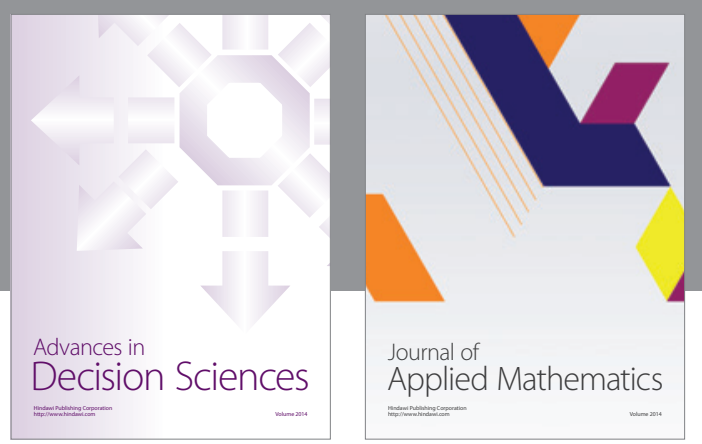

Algebra

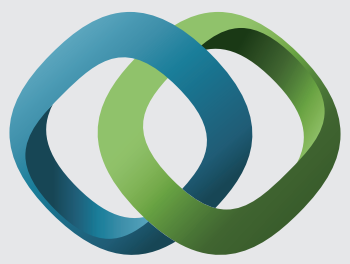

\section{Hindawi}

Submit your manuscripts at

http://www.hindawi.com
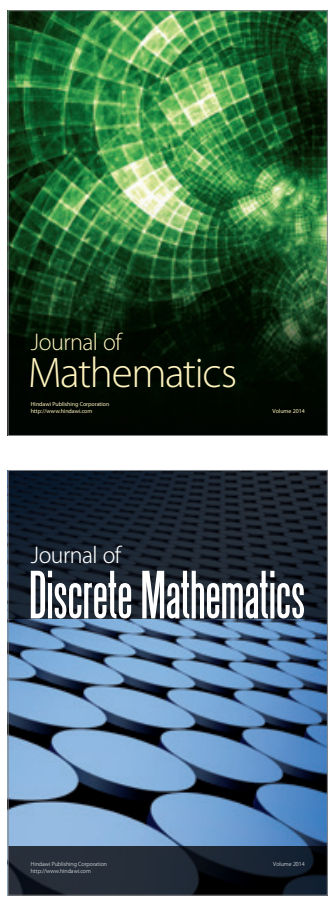

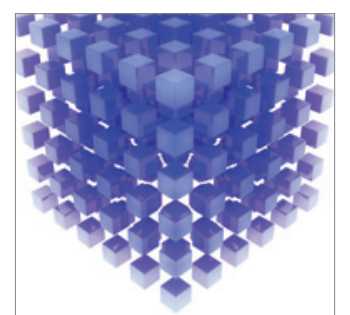

Mathematical Problems in Engineering
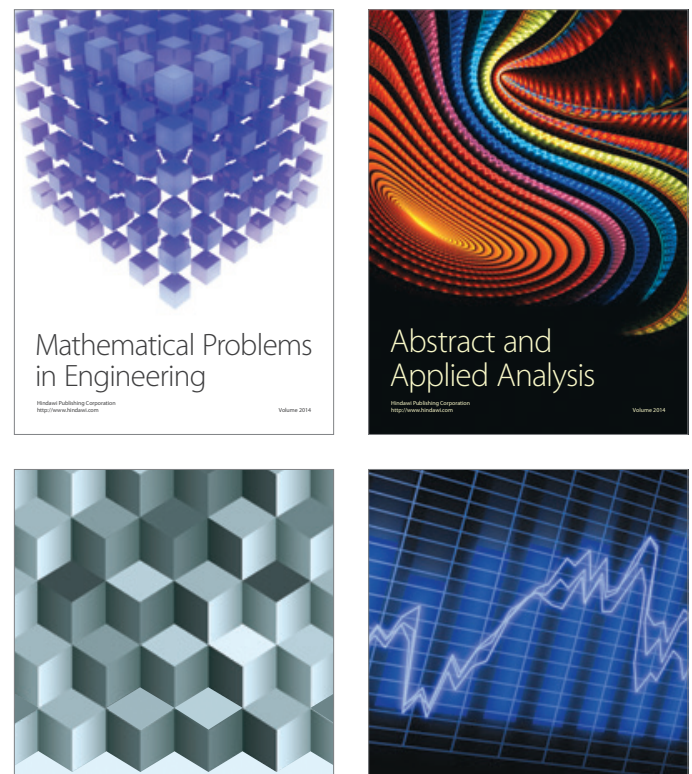

Journal of

Function Spaces

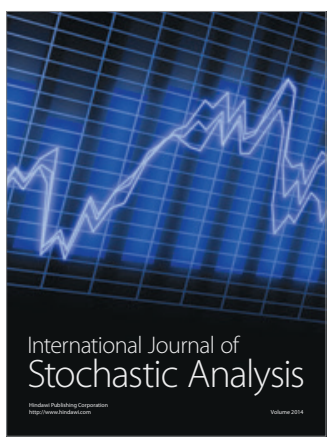

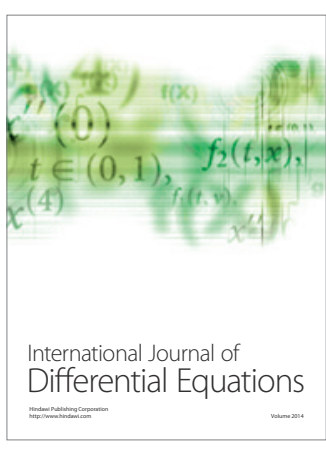
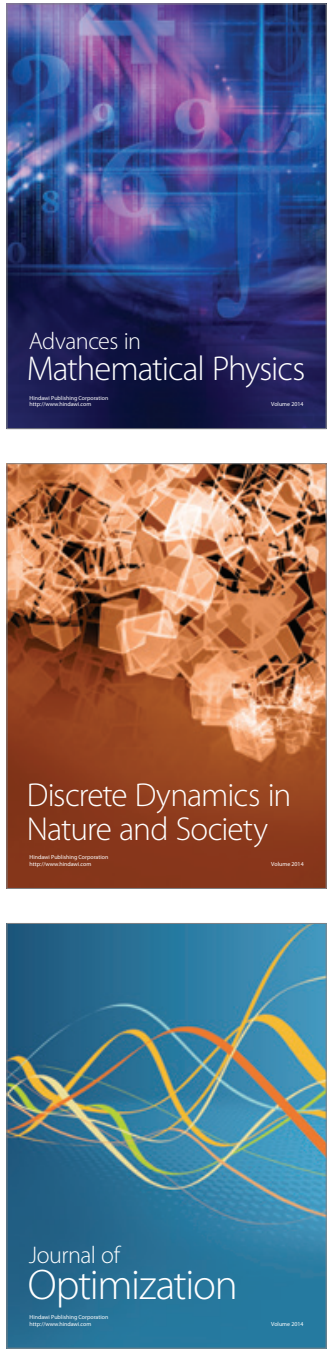